\title{
DIRECT HIS-BUNDLE PERMANENT PACING AS AN ALTERNATIVE TO CONVENTIONAL RIGHT VENTRICULAR APICAL PACING: A PILOT STUDY IN ZAGAZIG UNIVERSITY CARDIAC ELECTROPHYSIOLOGY LABORATORY.
}

\author{
Hisham S Roshdy \\ Cardiology department, Faculty of Medicine, Zagazig University, Zagazig, Egypt
}

\section{ABSTRACT}

Aim: was to evaluate the feasibility of direct his bundle pacing (DHBP) as one of the physiological pacing approaches in our EP lab.

Methods: All suitable patients admitted for permanent pacemaker implantation, between January and June 2013, were given a trial for DHBP. Using his bundle (HB) recording through a quadripolar catheter as an electrical and radiological guidance, custom shape stylet was used to direct an active fixation lead to the target position; a pacing protocol was applied to insure pure HB capture. A matching group of patients with apical right ventricular pacing (ARVP) were used as control, to assess the feasibility of DHBP technique.

Results: Pure DHBP was successful in 12 out of 32 patients $38 \%$. The DHBP procedure was costly when compared to ARVP in total procedure time ( $94.7 \pm 30.4$ vs. $43.2 \pm 17.4$ minutes), fluoroscopy time( $35.2 \pm 10.9$ vs. 10.1 \pm 3.6 minutes), patient $\mathrm{x}$-ray exposure $\left(34.1 \pm 7.8\right.$ vs. $\left.11.9 \pm 4.9 \mathrm{~Gy} \mathrm{Cm}^{2}\right)$ and acute pacing threshold $(2.1 \pm 0.6$ vs.0.6 $\pm 0.4 \mathrm{~V})$. But in the other hand it was better in paced QRS width (76.8 \pm 14.8 vs. $193.9 \pm 35.8 \mathrm{~ms}$.), patients' exercise capacity as percentage of achieved METS from Max METS for age $(75.5 \pm 8.7$ vs.54.9 $\pm 8.7 \%)$. the DHBP procedure time was progressively shorter indicating a steep learning curve.

Conclusion: Although DHBP has many technical difficulties, it can be achieved with a reasonable coast in our EP lab with a demonstrable benefit, and a steep learning curve.

Keywords: direct his bundle pacing, apical right ventricular pacing, physiological pacing, pacemaker, paced QRS duration, and active fixation lead.

\section{INTRODUCTION}

$\mathbf{T}$ he introduction of cardiac pacing in clinical practice led to logical concerns about the possible impact of the stimulation site on cardiac function. Key studies in the sixties, however, showed that atrial, right ventricular, and left ventricular pacing produced no short term differences in ventricular physiology either in patients with or without heart disease. These studies appeared to close the subject, and the problem of cardiac pacing was reduced to one of finding both identifying easiest access point which produced fewest complications. ${ }^{[1,2]}$ Two decades later, the technique was shown to be associated with several deleterious physiological effects, including asymmetrical ventricular hypertrophy, ventricular dilatation ${ }^{[3]}$, myofibrillar disarray ${ }^{[4]}$, increase in myocardial concentrations of catecholamines ${ }^{[5]}$, and alterations in myocardial perfusion ${ }^{[6]}$, the clinical deleterious effect was obvious in the DAVID ${ }^{[7]}$ and in the $\operatorname{MOST}^{[8]}$ trials. Non apical right ventricular pacing (ARVP) was extensively studied with no encouraging results, the ROVA study, did not show any improvement in quality of life or ventricular contraction when non apical pacing sites were used
[9]. If the aim is to mimic physiological activation patterns, the HB undoubtedly provides the ideal site. The first set of data comes from a team in Pennsylvania ${ }^{[10]}$, showed that Long-term, direct his pacing results in a reduction of left ventricular dimensions and improved cardiac function in patients with atrial fibrillation and dilated cardiomyopathy. During the last decade there was much work done in the field of DHBP, to extend the indications ${ }^{[11-16]}$ and to improve the technique ${ }^{[17-20]}$. But inspite of these efforts, DHBP is still great in theory, but difficult in practice ${ }^{[21]}$. The aim of this study was to assess the feasibility of direct DHBP in our EP lab using simple readily available techniques, and to compare the short term effect on exercise capacity of the patients.

\section{PATIENTS AND METHODS}

All patients admitted to cardiac EP lab, Zagazig University hospitals for permanent pacemaker implantation -according to the Guidelines for cardiac pacing and cardiac resynchronization therapy- ${ }^{[22]}$ between January and June 2013, were included in the study. A trial was made to implant a HB electrode, if the trial failed, the electrode was implanted in the right ventricular apex -according to 
our EP lab protocol for preparation and preforming single chamber pacemaker implantation- ${ }^{[23]}$. All patients were provided with VVIR pulse generators (with an activity sensor which is used to measure the patients' movement and to determine the appropriate pacing rate, programed at the same upper sensor rate). Patients with successful HB implantation were compared with a matching group of patients with an electrode implanted in the right ventricular (RV) apex. Signed informed consent was obtained from all patients. HB localization: HB recording was obtained using a quadripolar catheter with $2-5 \mathrm{~mm}$ electrode spacing introduced from the left femoral vein and advanced to the right atrium under fluoroscopy, the catheter was positioned in the anatomical position of the HB in the superior septal aspect of the TV, by advancing the catheter to the $\mathrm{RV}$ in right anterior oblique (RAO) projection and applying a clock wise torque and confirming the septal position in left anterior oblique (LAO) projection. Recording from the catheter is displayed on the cardiolab EP recording system (GE healthcare, WI, USA); electric stimulation was delivered to the catheter using EPS320 Cardiac Stimulator (Micropace Inc., CA, USA). If HB recording was obtained pacing was started at $4 \mathrm{v}$ at $50 \mathrm{~ms}$ pule width to capture the heart, 12 lead ECG simultaneously recorded should show a QRS width less than $120 \mathrm{~ms}$, or the output was reduced until loss of capture or QRS width reduced, if loss of capture occurred before reduction in QRS width, the catheter is slightly moved and the same pacing protocol is repeated. If no $\mathrm{HB}$ recording obtained the same pacing protocol is repeated. Once stable pacing is obtained at a pacing rate of $70 / \mathrm{min}$ the pacing rate was increased to $140 / \mathrm{min}$ and one to one conduction confirmed, the pacing threshold is determined, with $3 \mathrm{~V}$ or less at $50 \mathrm{~ms}$ is considered acceptable. A screw-in active fixation lead is used $52 \mathrm{~cm}$, active fixation Tendril SDX, St Jude leads (St Jude, Minneapolis, MN, USA), it was introduced through the left subclavian vein, a custom shaped style is preloaded in the lead with two curves, the first curve is big to access the superior aspect of the TV, and the second curve is smaller and orthogonal to the first one to access the septal aspect of the valve. The lead was manipulated to aim at the recording tip of the quadripolar catheter. To confirm the position of the pacing lead unipolar recording was obtained from the tip, and the previous pacing protocol was repeated using the pacing electrode. Once acceptable the screw was protruded to fix the lead in position. The pacemaker was programed to sense the patients QRS and not the $\mathrm{P}$ wave-if any- by adjusting the sensing threshold. Fluoroscopy time and exposure was noted, skin to skin time was noted. If the time from procedure start exceeded 120 minutes without achieving endpoints of paced narrow QRS in an acceptable pacing threshold the lead was fixed to the RV apex. After one month of implantation, an exercise ECG was done, to determine exercise capacity of the patients, measured in maximum metabolic equivalents (METS) which can be achieved by the patient, the percentage of METS achieved from the maximum calculated METS for age was calculate. Patients were excluded if they cannot exercise on a treadmill or having a comorbidity that may affect the exercise capacity. The patients were followed-up for 3 months to detect any complications. Statistical methods: All data were analyzed using SPSS software statistical package for social science version 16 (SPSS, Inc. Chicago, IL, USA). Results were presented as mean value $\pm \mathrm{SD}$ for continuous variables and as frequency $(\%)$ for categorical variables. Data were tested for normality using Kolmogorov-Smirnov test. Means were compared in independent groups using Student t-test or Mann-Whitney test.

\section{RESULTS}

Between January and June 2013, 83 pacemakers were implanted, among them, 32 patients were included in the study, with success to capture the HB achieved in 12 patients (38\%). Control group of 12 patients with matching demographic and clinical characteristics were selected from pacemaker clinic, there were four females in each group (36\%) $\mathrm{p}=0.67$. table 1 shows demographic and clinical characteristics of the 24 patients.

When we compared procedure time, DHBP procedure was twice as long as ARVP (218\%), fluoroscopy time was more than triple in DHBP pacing $(350 \%)$, patient exposure-using reduced sittings (low details, 7.5 frames/second)-was nearly triple ARVP (309\%), QRS width during DHBP was $40 \%$ of that during ARVP, pacing threshold in DHBP was 3.5 times higher than ARVP with the screw-in, which remained higher after one month and after 3 months, but followed the usual course of pacing threshold (initial elevation after one month and stabilization at a lower chronic pacing threshold later on), R wave amplitude in DHBP was $67 \%$ of 
the $\mathrm{R}$ wave amplitude in ARVP. There was $29 \%$ reduction of the pacing threshold when the screw was out, compared to the screw-in in the HB lead. As shown in table2.

After one month of implantation, calculated metabolic equivalents (METS) achieved by the patients, during symptom limited exercise stress test, was compared between the two groups, DHBP patients achieved more METS when compared to Max METS for the age as shown in table3.

Learning curve was steep, with improvement in the procedure duration over time, figure (1).

During the period of follow-up there were no cases of loss of capture or lead dislodgment.

Table1:demographic and, clinical and echocardiographic characteristics of the patients

\begin{tabular}{lcccc}
\hline & DHBP & ARVP & $\mathrm{t}$ & $\mathrm{P}$ \\
\hline Age (years) & $58.4 \pm 6.8$ & $59.3 \pm 8.2$ & -0.28 & 0.78 \\
\hline BMI & $28.5 \pm 3.5$ & $29.6 \pm 4.9$ & -0.54 & 0.59 \\
\hline Heamoglobin(gm/dl) & $11.4 \pm 1.8$ & $11.1 \pm 1.5$ & 0.61 & 0.43 \\
\hline LVEDV $(\mathrm{ml})$ & $84.9 \pm 12$. & $83.7 \pm 11$. & 0.24 & 0.81 \\
& 1 & 6 & & \\
\hline LVESV $(\mathrm{ml})$ & $31.5 \pm 7.6$ & $32.2 \pm 8.9$ & -0.18 & 0.85 \\
\hline EF $(\%)$ & $63.9 \pm 6.1$ & $61.9 \pm 8.1$ & 0.42 & 0.68 \\
\hline
\end{tabular}

Table2: Intra-procedural data comparison

\begin{tabular}{lllll}
\hline & \multicolumn{1}{c}{ DHBP } & \multicolumn{1}{c}{ ARVP } & t & P \\
\hline Procedure time (minutes) & $94.7 \pm 30.4$ & $43.2 \pm 17.4$ & 4.87 & $<0.001$ \\
\hline Fluoroscopy time (minutes) & $35.2 \pm 10.9$ & $10.1 \pm 3.6$ & 7.22 & $<0.001$ \\
\hline Patients exposure $\left.(\mathrm{Gy} \mathrm{cm})^{2}\right)$ & $34.1 \pm 7.8$ & $11.9 \pm 4.9$ & 7.94 & $<0.001$ \\
\hline QRS duration $(\mathrm{ms})$ & $76.8 \pm 14.8$ & $193.9 \pm 35.8$ & -10.0 & $<0.001$ \\
\hline Pacing threshold screw out(V) & $2.1 \pm 0.6$ & $0.6 \pm 0.4$ & 6.54 & $<0.001$ \\
\hline Pacing threshold screw in(V) & $1.5 \pm 0.5$ & & & \\
\hline Pacing threshold one month(V) & $2.3 \pm 1.3$ & $1.2 \pm 0.6$ & 3.56 & $<0.001$ \\
\hline Pacing threshold 3 months(V) & $1.2 \pm 0.7$ & $0.7 \pm 0.3$ & 4.46 & $<0.001$ \\
\hline R wave amplitude (mV) & $5.2 \pm 2.0$ & $7.7 \pm 1.9$ & -2.91 & $<0.001$ \\
\hline Lead impedance (ohms) & $361.4 \pm 55.1$ & $555.7 \pm 60.1$ & -7.84 & $<0.001$ \\
\hline
\end{tabular}

Table3: Exercise ECG data comparing both groups

\begin{tabular}{llllll}
\hline & DHBP & ARVP & t & P \\
\hline Max. METS for age & $10.2 \pm 0.68$ & $10.1 \pm 0.83$ & 0.27 & 0.74 & \\
\hline \% of achieved METS form MAX METS for age & $75.5 \pm 8.7$ & $54.9 \pm 8.7$ & 5.52 & $<0.001$ & \\
\hline
\end{tabular}




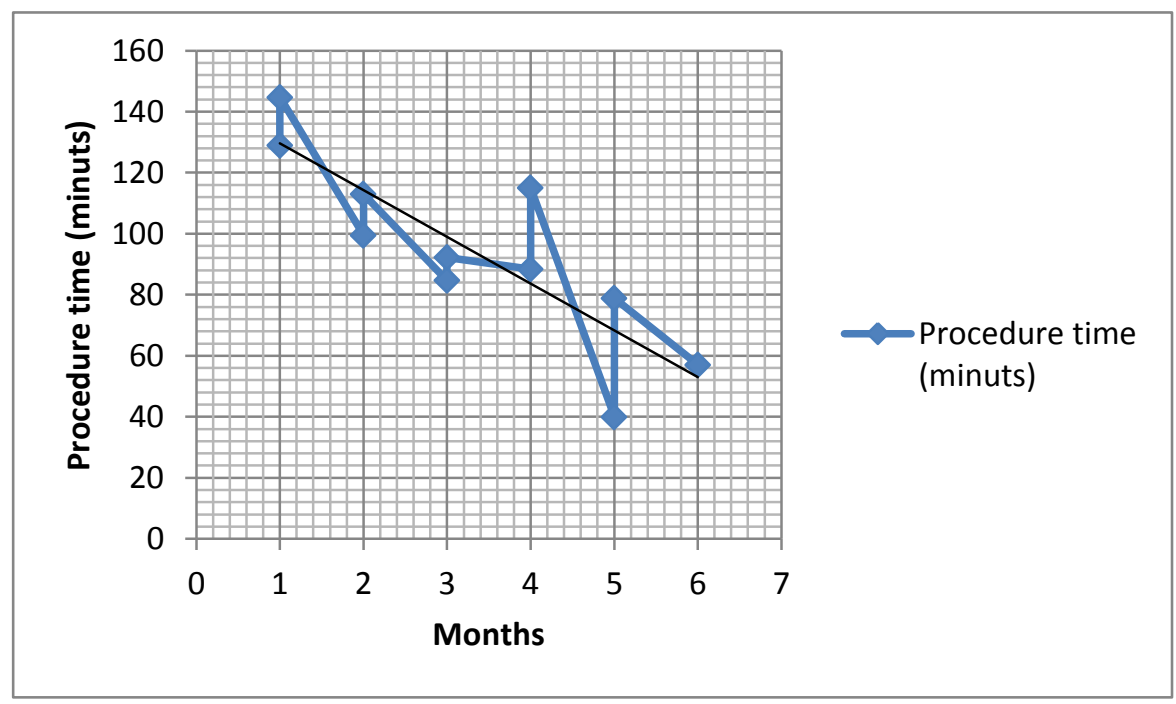

Figure (1): Linear regression plot showing the total procedure time of DHBP in each of the months of the study.

\section{DISCUSSION}

We succeed in $38 \%$ of our trials to establish a successful DHP, the most common cause of failure that we could not achieve a QRS width less than $120 \mathrm{~ms}$, the second most common cause was the inability to fix the lead in the target position, or the instability of the position, the third most common cause was the inability to maintain one to one conduction at a rate less than 140/minute. Deshmukh \& Romanyshyn ${ }^{[24]}$ used the same technique and achieved a success rate of $72 \%$ but they did not have a time limit, Cantu et al. ${ }^{[25]}$ achieved $64 \%$ success with using a special steerable catheter, Catanzariti et al. ${ }^{[26]}$ achieved $95 \%$ success using steerable catheter and coronary sinus catheter as a landmark, it seems that a well-equipped EP lab with experienced staff can have an essential rule in increasing the success rate, in the other hand, some of the previous authors considered a para-his pacing -with QRS wider than 120ms- as a successful HB pacing, which was not the case in our study, as we only accepted pure HB capture with QRS duration < $120 \mathrm{~ms}$.

Average total procedure time (skin to skin time) was $94.7 \pm 30.4$ vs. $43.2 \pm 17.4$ minutes and average fluoroscopy time was $35.2 \pm 10.9$ vs. $10.1 \pm 3.6$ minutes, for DHBP and ARVP respectively, The most significant challenge encountered was the inability to precisely direct the stylet-controlled lead tip to the small (2-mm diameter) target, especially during cardiac contraction and relaxation. Moreover, difficulty was frequently encountered when attempting to engage the screw into the membranous septum. Gross dislodgment of the lead tip requiring multiple attempts was a common occurrence that could be attributed to initial lack of experience and lack of active lead steering which necessitate many trials to refine the shape of the stylet and directing the tip of the electrode to a suitable position for pacing and fixation. Cantu et al. ${ }^{[25]}$ median fluoroscopy time was 16 minutes for DHBP and 17 minutes for para-his pacing. Catanzariti et al. [26] Mean procedural and fluoroscopy exposure times were $135.7 \pm 18.1 \mathrm{~min}$ (range $106-147$ ) and $10.2 \pm 4.1 \mathrm{~min}$, respectively, they used additional coronary catheter for land marking. Kronborg et al. ${ }^{[27]}$ Mean implantation time was $85 \pm 31 \mathrm{~min}$, mean fluoroscopy time was $23 \pm 13 \mathrm{~min}$, and mean position attempts of the HB lead was $8 \pm 5$. Total procedure time in our study was progressively shorter reflecting the steep learning curve of the procedure.

Pacing parameters were acceptable. As most of the authors set a target pacing threshold of $2.5 \mathrm{~V}$, we decided to increase it to $3 \mathrm{~V}$ as the first cases showed a good capture between $3 \mathrm{~V}$ and $2.5 \mathrm{~V}$ but not below $2.5 \mathrm{~V}$, which was reduced when the screw was protruded. Cantu et al. ${ }^{[25]}$ reported nearly similar parameters; QRS duration was $99 \pm 19 \mathrm{~ms}$. the acute $\mathrm{HB}$ threshold at $0.5 \mathrm{~ms}$ was $1.7 \pm 1.2 \mathrm{v}$. Catanzariti et 
al. ${ }^{[26]}$ Pacing thresholds, impedance and sensed potentials of the HB lead, recorded 30-60 min after lead fixation and at the last follow-up examination, were $1.61 \pm 0.55$ and $1.71 \pm 0.92 \mathrm{~V}$ at a pulse width of $0.5 \mathrm{~ms}, 552 \pm 89$ and $525 \pm 101 \mathrm{ohm}, 4.32 \pm 2.77$ and $4.17 \pm 3.63 \mathrm{~V}$, respectively. The pacing threshold In our study dropped to similar limits after fixation of the lead, but we preferred to report the prefixation values to try making the procedure faster, by skipping many trials of fixing and unfixing the lead, the chronic pacing threshold was nearly the same as the acute pacing threshold, with the usual elevation at one month of implantation, in some of our patients, we had to increase the factory default pacing voltage to achieve a safety margin of double the pacing threshold.

The marked improvement in exercise capacity observed using symptom limited exercise testing to measure the Max. achievable METS and correcting it as a percentage of the MAX predicted METS for age was agreed by Deshmukh \& Romanyshyn ${ }^{[24]}$ using the LV pressure-volume curves recorded improvement in patient $s$ with DHBP, in the same study there was a marked improvement in cardiopulmonary testing parameters in patients with DHBP vs. ARVP.in the other hand Padeletti et al. ${ }^{[28]}$ demonstrated no improvement in LV pressurevolume curves when measured during acute pacing from different sites including DHBP. in the first study the patients baseline EF was much lower than the second study, furthermore the second study adopted only the acute effect of pacing, while the firs one like in our case demonstrated the chronic effect of HB pacing, and its expected-as proved by the first study- that increase in the stock volume with less oxygen consumption is the direct cause of increased exercise capacity even in patients with normal LV systolic function.

\section{CONCLUSION}

In conclusion, this study demonstrated the difficulties accompanied with DHBP, in the other hand the great benefits gained from this approach including; avoiding the established deleterious ARVP; and the acute gain in exercise capacity, which will be reflected as an improvement in the life style

\section{STUDY LIMITATIONS}

The small number in addition to the short period of follow-up was the main limitations of this study, but we considered it as a pilot study to test the acute feasibility of the DHBP in our EP lab. The lack of hemodynamic confirmation of superiority of the DHBP to ARVP was another limitation, but we are planning to include a larger number of patients in a study which will include invasive and non-invasive hemodynamic comparison.

\section{REFERENCES}

1. Fletcher FW, EO Theilen, MS Lawrence, et al. (1963). "Effect of Pacemaker Location on Cardiac Function in Complete a-V Heart Block". Am J Physiol. 205: p. 1232-4.

2. Benchimol A and MS Liggett (1966). "Cardiac hemodynamics during stimulation of the right atrium, right ventricle, and left ventricle in normal and abnormal hearts". Circulation. 33(6): p. 933-44.

3. van Oosterhout MF, FW Prinzen, T Arts, et al. (1998). "Asynchronous electrical activation induces asymmetrical hypertrophy of the left ventricular wall". Circulation. 98(6): p. 588-95.

4. Adomian GE and J Beazell (1986). "Myofibrillar disarray produced in normal hearts by chronic electrical pacing". Am Heart J. 112(1): p. 79-83.

5. Lee MA, MW Dae, JJ Langberg, et al. (1994). "Effects of long-term right ventricular apical pacing on left ventricular perfusion, innervation, function and histology". J Am Coll Cardiol. 24(1): p. 225-32.

6. Tse HF and CP Lau (1997). "Long-term effect of right ventricular pacing on myocardial perfusion and function". J Am Coll Cardiol. 29(4): p. 744-9.

7. Wilkoff BL, JR Cook, AE Epstein, et al. (2002). "Dual-chamber pacing or ventricular backup pacing in patients with an implantable defibrillator: the Dual Chamber and VVI Implantable Defibrillator (DAVID) Trial". JAMA. 288(24): p. 3115-23.

8. Sweeney MO, AS Hellkamp, KA Ellenbogen, et al. (2003). "Adverse effect of ventricular pacing on heart failure and atrial fibrillation among patients with normal baseline QRS duration in a clinical trial of pacemaker therapy for sinus node dysfunction". Circulation. 107(23): p. 2932-7.

9. Stambler BS, K Ellenbogen, X Zhang, et al. (2003). "Right ventricular outflow versus apical pacing in pacemaker patients with congestive heart failure and atrial fibrillation". J Cardiovasc Electrophysiol. 14(11): p. 1180-6.

10. Deshmukh P, DA Casavant, M Romanyshyn, et al. (2000). "Permanent, direct his-bundle pacing - A novel approach to cardiac pacing in patients with normal His-purkinje activation". Circulation. 101(8): p. 869-877.

11. Kronborg MB, SH Poulsen, PT Mortensen, et al. (2012). "Left ventricular performance during paraHis pacing in patients with high-grade atrioventricular block: an acute study". Europace. 14(6): p. 841-846. 
12. Rehwinkel AE, JG Muller, PC Vanburen, et al. (2011). "Ventricular Resynchronization by Implementation of Direct His Bundle Pacing in a Patient with Congenital Complete AV Block and Newly Diagnosed Cardiomyopathy". Journal of Cardiovascular Electrophysiology. 22(7): p. 818821.

13. Manovel A, R Barba-Pichardo, and A Tobaruela (2011). "Electrical and Mechanical Cardiac Resynchronisation by Novel Direct His-bundle Pacing in a Heart Failure Patient". Heart Lung and Circulation. 20(12): p. 769-772.

14. Barba-Pichardo R, P Morina-Vazquez, J VenegasGamero, et al. (2006). "Permanent his-bundle pacing in patients with infra-Hisian atrioventricular block". Revista Espanola De Cardiologia. 59(6): p. 553-558

15. Kirkutis A, A Poviliunas, P Griciene, et al. (2004). "Cardiac rate normalization in chronic atrial fibrillation: Comparison of long-term efficacy of treatment with amiodarone versus AV node ablation and permanent his-bundle pacing". Proceedings of the Western Pharmacology Society. p. 69-70.

16. Vazquez PM, RB Pichardo, JV Gamero, et al. (2001). "Permanent pacing of the His bundle after radiofrequency atrioventricular node ablation in patients with suprahisian conduction disturbances". Revista Espanola De Cardiologia. 54(12): p. 13851393.

17. Barba-Pichardo R, AM Sanchez, JM FernandezGomez, et al. (2013). "Ventricular resynchronization therapy by direct His-bundle pacing using an internal cardioverter defibrillator". Europace. 15(1): p. 83-88.

18. Yin LX, TG Laske, N Rakow, et al. (2008). "Intracardiac echocardiography-guided his bundle pacing and atrioventricular nodal ablation". PacePacing and Clinical Electrophysiology. 31(5): p. 536-542.

19. Tung S, K Rajagopalan, J Affolter, et al. (2008). "Unipolar Pace Mapping With the Medtronic SelectSecure 3830 Lead System can Accurately Locate the HIS Bundle for Permanent HIS Bundle Pacing". Circulation. 118(18): p. S680-S681.
20. Zanon F, E Baracca, S Aggio, et al. (2006). "A feasible approach for direct His-bundle pacing using a new steerable catheter to facilitate precise lead placement". Journal of Cardiovascular Electrophysiology. 17(1): p. 29-33.

21. Garrote JA (2006). "His-bundle pacing: Great concept, difficult in practice". Revista Espanola De Cardiologia. 59(6): p. 534-536.

22. Vardas PE, A Auricchio, JJ Blanc, et al. (2007). "Guidelines for cardiac pacing and cardiac resynchronization therapy. The Task Force for Cardiac Pacing and Cardiac Resynchronization Therapy of the European Society of Cardiology. Developed in collaboration with the European Heart Rhythm Association". Europace. 9(10): p. 959-98.

23. Kusumoto FM (2007)"Cardiac pacing for the clinician". Springer-Norwell: New York. p. 107246.

24. Deshmukh PM and M Romanyshyn (2004). "Direct his-bundle pacing: Present and future". Pace-Pacing and Clinical Electrophysiology. 27(6): p. 862-870.

25. Cantu F, P De Filippo, P Cardano, et al. (2006). "Validation of criteria for Selective His Bundle and para-Hisian permanent pacing". Pace-Pacing and Clinical Electrophysiology. 29(12): p. 1326-1333.

26. Catanzariti D, M Maines, C Cemin, et al. (2006). "Permanent direct his bundle pacing does not induce ventricular dyssynchrony unlike conventional right ventricular apical pacing - An intrapatient acute comparison study". Journal of Interventional Cardiac Electrophysiology. 16(2): p. 81-92.

27. Kronborg MB, PT Mortensen, JC Gerdes, et al. (2011). "His and para-His pacing in AV block: feasibility and electrocardiographic findings". Journal of Interventional Cardiac Electrophysiology. 31(3): p. 255-262.

28. Padeletti L, R Lieberman, J Schreuder, et al. (2007). "Acute effects of his bundle pacing versus left ventricular and right ventricular pacing on left ventricular function". American Journal of Cardiology. 100(10): p. 1556-1560. 
تنظيم القلب الدائم عن طريق ضفيرة هس كبديل عن التظيم التقليدي من قمة البطين الايمن: دراسة تجريبية في مختبركهروفسيلوجية القلب بجامعة الزقازيق من البط

\author{
هثام سمير رشدي الطندي \\ قسم القلب و الاو عية الاموية، كلية الظّب جامعة الزقازيق
}

يعد تنظيم القلب فسيولوجيا (اقرب الي التنظيم الطبيعي) من أهداف اختصاصيو كهربية القلب، وخصوصا بعد التقارير المبكره عن الاثار الضسارة

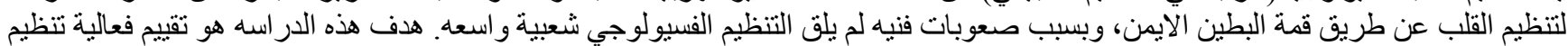
القلب عن طريق ضفيرة هس.

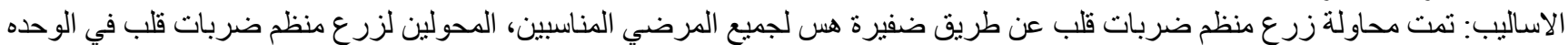

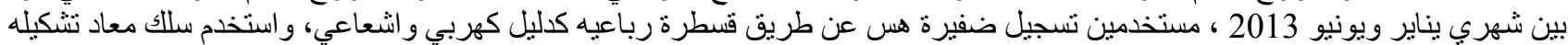

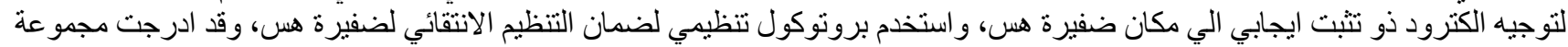

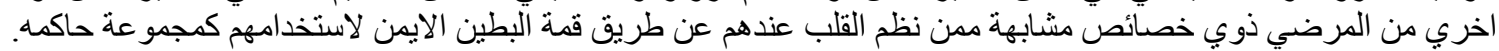

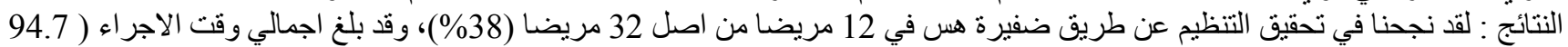

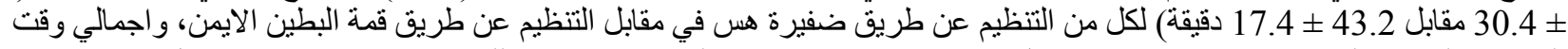

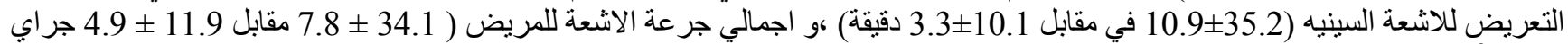

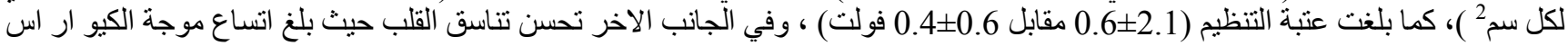

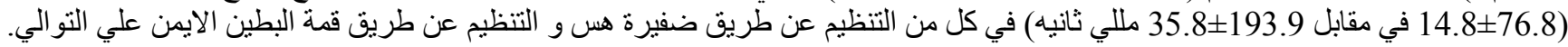
كما وصلت نسبة ما يستطيع المريض تحقيقه من مكافئات الجها منسوبة الي الحدود القصوي بالنسبة للسن (

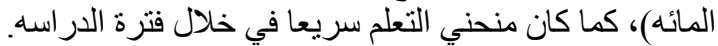

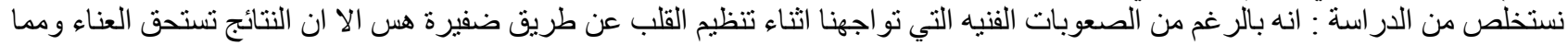
يشجع علي الاستمر ار في هذا النهج منحني التعلم السريع لهذا الاجر ألتهاء. 